Article

\title{
Mean Value of the General Dedekind Sums over Interval $\left[1, \frac{q}{p}\right)$
}

\section{Lei Liu and Zhefeng $\mathrm{Xu}$ *}

School of Mathematics, Northwest University Xi'an, Xi'an 710069, China; liulei@stumail.nwu.edu.cn

* Correspondence: zfxu@nwu.edu.cn

Received: 4 November 2020; Accepted: 11 December 2020; Published: 15 December 2020

Abstract: Let $q>2$ be a prime, $p$ be a given prime with $p<q$. The main purpose of this paper is using transforms, the hybrid mean value of Dirichlet $L$-functions with character sums and the related properties of character sums to study the mean value of the general Dedekind sums over interval $\left[1, \frac{q}{p}\right)$, and give some interesting asymptotic formulae.

Keywords: general Dedekind sum; character sums; L-functions; mean value

\section{Introduction}

For a positive integer $k$ and an arbitrary integer $h$ with $(h, k)=1$, the classical Dedekind sum $S(h, k)$ is defined by

$$
S(h, k)=\sum_{a=1}^{k}\left(\left(\frac{a}{k}\right)\right)\left(\left(\frac{a h}{k}\right)\right)
$$

where

$$
((x))= \begin{cases}x-[x]-\frac{1}{2}, & \text { if } x \text { not be an integer; } \\ 0, & \text { if } x \text { be an integer. }\end{cases}
$$

It plays a significant role in the transformation theory of the Dedekind $\eta$ function. In [1-5], many researchers have investigated the various properties of $S(h, k)$. Perhaps the most well-known property of the Dedekind sums is the reciprocity formula

$$
S(h, k)+S(k, h)=\frac{h^{2}+k^{2}+1}{12 h k}-\frac{1}{4} .
$$

Conrey, J.B. et al. [2] studied the $2 m$-th power mean of $S(h, k)$, and proved the following important asymptotic formula

$$
\sum_{h=1}^{k} S^{2 m}(h, k)=f_{m}(k)\left(\frac{k}{12}\right)^{2 m}+O\left(\left(k^{9 / 5}+k^{2 m-1+1 /(m+1)}\right) \log ^{3} k\right),
$$

where $\sum_{h}^{\prime}$ denotes summation over all $h$ such that $(h, k)=1$ and $f_{m}(k)$ is defined by the Dirichlet series

$$
\sum_{k=1}^{\infty} \frac{f_{m}(k)}{k^{s}}=2 \frac{\zeta^{2}(2 m)}{\zeta(4 m)} \cdot \frac{\zeta(s+4 m-1)}{\zeta^{2}(s+2 m)} \cdot \zeta(s) .
$$

For $m \geq 2$, Jia, C. [3] reduced the error terms to $O\left(k^{2 m-1}\right)$. While for $m=1$, Zhang, W. [5] showed

$$
\sum_{h=1}^{k} S^{2}(h, k)=\frac{5}{144} k \phi(k) \prod_{p^{\alpha} \| k}\left(1+\frac{1}{p}+\frac{1}{p^{2}}\right)^{-1}\left[\left(1+\frac{1}{p}\right)^{2}-\frac{1}{p^{3 \alpha+1}}\right]+O\left(k \exp \left(\frac{4 \log k}{\log \log k}\right)\right) .
$$


Zhang, W. and Yi, Y. [6] studied the first mean value of $S(h, k)$, and obtained an asymptotic formula

$$
\sum_{n \leq N}^{\prime} S(n, k)=\frac{1}{12} \phi(k)\left(\log N+\gamma+\sum_{p \mid k} \frac{\log p}{p-1}\right)+O\left(\frac{k 2^{\omega(k)}}{N}+N k^{\epsilon}\right)
$$

for positive integer $k$ and $1<N \leq \frac{1}{2} k$.

Zhang, W. [7] defined the general Dedekind sum $S(h, n, k)$ as follows:

$$
S(h, n, k)=\sum_{a=1}^{k} \overline{B_{n}}\left(\frac{a}{k}\right) \overline{B_{n}}\left(\frac{a h}{k}\right),
$$

where

$$
\overline{B_{n}}(x)= \begin{cases}B_{n}(x-[x]), & \text { if } x \text { is not an integer } \\ 0, & \text { if } x \text { is an integer. }\end{cases}
$$

called the $n$-th periodic Bernoulli polynomials defined on $0<x \leq 1$, and $B_{n}(x)$ is the $n$-th Bernoulli polynomials. Clearly, $S(h, 1, k)=S(h, k)$ is the classical Dedekind sum.

Recently, Kim et al. [8-10] studied the poly-Dedekind sums given by

$$
S_{n}^{(m)}(h, k)=\sum_{a=1}^{k}\left(\frac{a}{k}\right) \bar{B}_{n}^{(m)}\left(\frac{a h}{k}\right),
$$

where $\bar{B}_{n}^{(m)}(x)=B_{n}^{(m)}(x-[x])$ are the type 2 poly-Bernoulli functions of index $m$, and obtained some interesting identities. Obviously, $S_{1}^{(1)}=S(h, k)$ is the classical Dedekind sum.

Let $q>2$ be a prime, $p$ be a given prime with $p<q$. Using the similar method of Shparlinski, I.E. [11] and combining with the mean value of $L$-functions and estimate of character sums, the authors and Wang, N. [12] studied the mean value distribution of the general Dedekind sums over short interval, that is

$$
\sum_{a \leq N} \sum_{b \leq N} a^{l} b^{k} S(a \bar{b}, n, q),
$$

here $n$ and $N$ be two positive integers with $q^{\epsilon} \leq N \leq q^{1-\epsilon}, l, k$ be two non-negative integers and $\bar{b}$ denote the multiplicative inverse of $b$ modulo $q$. However, in the final remarks, Shparlinski, I.E. [11] pointed out that "the author sees no reason why an appropriate asymptotic formula cannot hold for even larger values of $N$, up to $q / 2$ ". In this paper we can take $N$ to $q / p$, then through transform, mean value of Dirichlet $L$-functions and the properties of character sums to study the mean value of the general Dedekind sums over interval $\left[1, \frac{q}{p}\right)$, and obtain some sharper asymptotic formulae for it.

Now we give the main conclusion.

Theorem 1. Let $q>2$ be a prime, $p$ be a given prime with $p<q, n$ be a positive integer. Then we have

(i) when $n$ be an even number,

$$
\sum_{a<\frac{q}{p}} \sum_{b<\frac{q}{p}} S(a \bar{b}, n, q)=\frac{(n !)^{2} q^{2}}{2^{2 n-2} \pi^{2 n}}\left(\frac{1}{2 \pi^{2}} C_{p, n}-\frac{\zeta^{2}(n)}{p^{2}}\right)+O\left(q^{1+\epsilon}\right),
$$

here $C_{p, n}=\sum_{u=1}^{\infty} \frac{\gamma_{p}^{2}(u, n)}{u^{2}}, \gamma_{p}(u, n)=\sum_{d_{1} d_{2}=u} \sin \frac{2 \pi d_{1}}{p} \cdot d_{2}^{1-n}$

(ii) when $n$ be an odd number,

$$
\sum_{a<\frac{q}{p}} \sum_{b<\frac{q}{p}} S(a \bar{b}, n, q)=\frac{(n !)^{2} q^{2}}{2^{2 n-2} \pi^{2 n+2}} T_{p, n}+O\left(q^{1+\epsilon}\right)
$$


here

$$
\begin{aligned}
& T_{p, n}=\quad \frac{1}{2} \sum_{u=1}^{\infty} \frac{v_{p}^{2}(u, n)}{u^{2}}+\frac{1}{2}\left(1+\frac{1}{p^{2}}\right) \sum_{u=1}^{\infty} \frac{v^{2}(u, n)}{u^{2}} \\
& +\frac{1}{p^{2}} \sum_{u=1}^{\infty} \frac{v(u, n) v_{p}(p u, n)}{u^{2}}-\frac{1}{p^{2}} \sum_{u=1}^{\infty} \frac{v(u, n) v(p u, n)}{u^{2}} \\
& -\sum_{u=1}^{\infty} \frac{v(u, n) v_{p}(u, n)}{u^{2}} \\
& v_{p}(u, n)=\sum_{d_{1} d_{2}=u} \cos \frac{2 \pi d_{1}}{p} \cdot d_{2}^{1-n}, v(u, n)=\sum_{d \mid u} d^{1-n} .
\end{aligned}
$$

It is clear that $C_{p, n}$ and $T_{p, n}$ are constants depending on $p$ and $n$. From our theorem we may immediately deduce the following corollaries:

Corollary 1. Let $q>2$ be a prime, we have

$$
\begin{aligned}
& \sum_{a<\frac{q}{2}} \sum_{b<\frac{q}{2}} S(a \bar{b}, 2, q)=-\frac{q^{2}}{144}+O\left(q^{1+\epsilon}\right), \\
& \sum_{a<\frac{q}{2}} \sum_{b<\frac{q}{2}} S_{2}^{(1)}(a \bar{b}, q)=\frac{\pi}{144 i} q^{2}+O\left(q^{1+\epsilon}\right) .
\end{aligned}
$$

Corollary 2. Let $q>2$ be a prime, we have

$$
\begin{aligned}
& \sum_{a<\frac{q}{2}} \sum_{b<\frac{q}{2}} S(a \bar{b}, 4, q)=-\frac{q^{2}}{3600}+O\left(q^{1+\epsilon}\right), \\
& \sum_{a<\frac{q}{2}} \sum_{b<\frac{q}{2}} S_{4}^{(1)}(a \bar{b}, q)=\frac{\pi^{3} i}{10800} q^{2}+O\left(q^{1+\epsilon}\right) .
\end{aligned}
$$

For the general index $m$, the method of our article does not obtain the expected result. It would be an interesting question to continue to study the mean value of $S_{n}^{(m)}(h, k)$.

\section{Some Lemmas}

To prove the theorem, We need the following lemmas.

Lemma 1. Let $k$ and $r$ be integers with $k \geq 2$ and $(r, k)=1, \chi$ be a Dirichlet character modulo $k$. Then we have

$$
\sum_{\chi \bmod k}^{*} \chi(r)=\sum_{d \mid(k, r-1)} \mu\left(\frac{k}{d}\right) \phi(d)
$$

where $\Sigma^{*}{ }_{\chi \bmod k}$ denotes the summation over all primitive characters modulo $k$.

Proof. See Lemma 4 of reference [13].

Lemma 2. Let $k \geq 3$ and $h$ be two integers with $(h, k)=1, n$ be positive integer. Then we have

$$
S(h, n, k)=\frac{(n !)^{2}}{4^{n-1} k^{2 n-1} \pi^{2 n}} \sum_{d \mid k} \frac{d^{2 n}}{\phi(d)} \sum_{\substack{\chi \bmod d \\ \chi(-1)=-1}} \chi(h)|L(n, \chi)|^{2}
$$


for odd $n$ and

$$
S(h, n, k)=\frac{(n !)^{2}}{4^{n-1} k^{2 n-1} \pi^{2 n}} \sum_{d \mid k} \frac{d^{2 n}}{\phi(d)} \sum_{\substack{\chi \bmod d \\ \chi(-1)=1}} \chi(h)|L(n, \chi)|^{2}-\frac{(n !)^{2}}{4^{n-1} \pi^{2 n}} \zeta^{2}(n)
$$

for even $n$.

Proof. See Theorem of reference [7].

Lemma 3. Let $\chi$ be a primitive Dirichlet character modulo $k$. Then for any real number $\lambda \in[0,1)$ with $\lambda \neq \frac{r}{k}$, we have

$$
\sum_{a=1}^{[\lambda k]} \chi(a)= \begin{cases}\frac{\tau(\chi)}{\pi} \sum_{n=1}^{\infty} \frac{\bar{\chi}(n) \sin (2 \pi n \lambda)}{n}, & \text { if } \chi(-1)=1 \\ \frac{\tau(\chi)}{\pi i} \sum_{n=1}^{\infty} \frac{\bar{\chi}(n)(1-\cos (2 \pi n \lambda))}{n}, & \text { if } \chi(-1)=-1\end{cases}
$$

where $[x]$ denotes the greatest integer less than or equal to $x, \tau(\chi)=\sum_{a=1}^{k} \chi(a) e\left(\frac{a}{k}\right)$ is the Gauss sum and $e(y)=e^{2 \pi i y}$.

Proof. See Section 3.1 of [14].

Lemma 4. Let $k>3$ be an integer and $p$ be a prime with $p \nmid k$ and $p<k$. Then we have the identity (i) for even primitive character $\chi \bmod k$,

$$
\sum_{a<\frac{k}{p}} \chi(a)=\frac{\tau(\chi)}{\pi i \phi(p)} \sum_{\substack{\xi \bmod p \\ \xi(-1)=-1}} \tau(\bar{\xi}) L(1, \xi \bar{\chi})
$$

(ii) for odd primitive character $\chi \bmod k$,

$$
\sum_{a<\frac{k}{p}} \chi(a)=\frac{\tau(\chi)}{\pi i}\left(1-\frac{\bar{\chi}(p)}{p}\right) L(1, \bar{\chi})+\frac{i \tau(\chi)}{\pi \phi(p)} \sum_{\substack{\xi \bmod p \\ \xi(-1)=1}} \tau(\bar{\xi}) L(1, \xi \bar{\chi}) .
$$

Proof. From Lemma 3, (i) when $\chi(-1)=1$, we can write

$$
\sum_{a<\frac{k}{p}} \chi(a)=\frac{\tau(\chi)}{\pi} \sum_{n=1}^{\infty} \bar{\chi}(n) \frac{\sin (2 \pi n / p)}{n}=\frac{\tau(\chi)}{\pi} \sum_{h=1}^{p-1} \sin \frac{2 \pi h}{p} \sum_{\substack{n=1 \\ n \equiv h(\bmod p)}}^{\infty} \frac{\bar{\chi}(n)}{n} .
$$

Now

$$
\sum_{\substack{n=1 \\ n \equiv h(\bmod p)}}^{\infty} \frac{\bar{\chi}(n)}{n}=\frac{1}{\phi(p)} \sum_{\xi \bmod p} \bar{\xi}(h) \sum_{n=1}^{\infty} \frac{\xi \bar{\chi}(n)}{n}=\frac{1}{\phi(p)} \sum_{\xi \bmod p} \bar{\xi}(h) L(1, \bar{\xi} \bar{\chi}) .
$$

Furthermore,

$$
\begin{aligned}
\sum_{h=1}^{p-1} \bar{\xi}(h) \sin \frac{2 \pi h}{p} & =\frac{1}{2 i} \sum_{h=1}^{p-1} \bar{\xi}(h)\left(e\left(\frac{h}{p}\right)-e\left(\frac{-h}{p}\right)\right) \\
& =\frac{1}{2 i}(1-\xi(-1)) \tau(\bar{\xi}),
\end{aligned}
$$

where $e(x)=e^{2 \pi i x}$. 
Thus, we obtain the identity for $\chi(-1)=1$,

$$
\sum_{a<\frac{k}{p}} \chi(a)=\frac{\tau(\chi)}{\pi i \phi(p)} \sum_{\substack{\xi \bmod p \\ \xi(-1)=-1}} \tau(\bar{\xi}) L(1, \xi \bar{\chi})
$$

(ii) when $\chi(-1)=-1$, we can write

$$
\begin{aligned}
\sum_{a<\frac{k}{p}} \chi(a) & =\frac{\tau(\chi)}{\pi i} \sum_{n=1}^{\infty} \bar{\chi}(n) \frac{1-\cos (2 \pi n / p)}{n} \\
& =\frac{\tau(\chi)}{\pi i}\left(L(1, \bar{\chi})-\sum_{n=1}^{\infty} \bar{\chi}(n) \frac{\cos (2 \pi n / p)}{n}\right) \\
& =\frac{\tau(\chi)}{\pi i} L(1, \bar{\chi})+\frac{\tau(\chi)}{\pi} \sum_{n=1}^{\infty} \bar{\chi}(n) \frac{i \cos (2 \pi n / p)}{n}
\end{aligned}
$$

Now

$$
\begin{aligned}
& \sum_{n=1}^{\infty} \bar{\chi}(n) \frac{i \cos (2 \pi n / p)}{n} \\
= & i \sum_{h=1}^{p-1} \cos \frac{2 \pi h}{p} \sum_{\substack{n=1 \\
n \equiv h(\bmod p)}}^{\infty} \frac{\bar{\chi}(n)}{n} \\
= & i \sum_{h=1}^{p-1} \cos \frac{2 \pi h}{p} \cdot \frac{1}{\phi(p)} \sum_{\xi \bmod p} \bar{\xi}(h) L(1, \bar{\xi} \bar{\chi})+\frac{i \bar{\chi}(p)}{p} L(1, \bar{\chi}) \\
= & \frac{i}{\phi(p)} \sum_{\xi \bmod p}\left(\sum_{h=1}^{p-1} \bar{\xi}(h) \cos \frac{2 \pi h}{p}\right) L(1, \bar{\zeta} \bar{\chi})+\frac{i \bar{\chi}(p)}{p} L(1, \bar{\chi}),
\end{aligned}
$$

noting that

$$
e\left(\frac{h}{p}\right)+e\left(\frac{-h}{p}\right)=2 \cos \frac{2 \pi h}{p}
$$

we have

$$
\sum_{h=1}^{p-1} \bar{\xi}(h) \cos \frac{2 \pi h}{p}=\frac{1}{2} \sum_{h=1}^{p-1} \bar{\xi}(h)\left[e\left(\frac{h}{p}\right)+e\left(\frac{-h}{p}\right)\right]=\frac{1+\bar{\xi}(-1)}{2} \tau(\bar{\xi}) .
$$

So, we can get

$$
\sum_{n=1}^{\infty} \bar{\chi}(n) \frac{i \cos (2 \pi n / p)}{n}=\frac{i}{\phi(p)} \sum_{\substack{\xi \bmod p \\ \xi(-1)=1}} \tau(\bar{\xi}) L(1, \xi \bar{\chi})+\frac{i \bar{\chi}(p)}{p} L(1, \bar{\chi}) .
$$

Thus, we obtain the identity for $\chi(-1)=-1$,

$$
\sum_{a<\frac{k}{p}} \chi(a)=\frac{\tau(\chi)}{\pi i}\left(1-\frac{\bar{\chi}(p)}{p}\right) L(1, \bar{\chi})+\frac{i \tau(\chi)}{\pi \phi(p)} \sum_{\substack{\xi \bmod p \\ \xi(-1)=1}} \tau(\bar{\xi}) L(1, \xi \bar{\chi}) .
$$

This proves Lemma 4. 
Lemma 5. Let $q>2$ be a prime, $p$ be a given prime with $p<q, n$ be a positive integer. Then we have

$$
\sum_{a<\frac{q}{p}} \sum_{b<\frac{q}{p}} \sum_{\chi \bmod q} \chi(a \bar{b})|L(n, \chi)|^{2}=\frac{q^{2}}{2 \pi^{2}} C_{p, n}+O\left(q^{1+\epsilon}\right),
$$

here $C_{p, n}=\sum_{u=1}^{\infty} \frac{\gamma_{p}^{2}(u, n)}{u^{2}}, \gamma_{p}(u, n)=\sum_{d_{1} d_{2}=u} \sin \frac{2 \pi d_{1}}{p} \cdot d_{2}^{1-n}$. and

$$
\sum_{a<\frac{q}{p}} \sum_{b<\frac{q}{p}} \sum_{\substack{\chi \bmod q \\ \chi(-1)=-1}} \chi(a \bar{b})|L(n, \chi)|^{2}=\frac{q^{2}}{\pi^{2}} T_{p, n}+O\left(q^{1+\epsilon}\right),
$$

here

$$
\begin{aligned}
& T_{p, n}= \frac{1}{2} \sum_{u=1}^{\infty} \frac{v_{p}^{2}(u, n)}{u^{2}}+\frac{1}{2}\left(1+\frac{1}{p^{2}}\right) \sum_{u=1}^{\infty} \frac{v^{2}(u, n)}{u^{2}} \\
&+\frac{1}{p^{2}} \sum_{u=1}^{\infty} \frac{v(u, n) v_{p}(p u, n)}{u^{2}}-\frac{1}{p^{2}} \sum_{u=1}^{\infty} \frac{v(u, n) v(p u, n)}{u^{2}} \\
&-\sum_{u=1}^{\infty} \frac{v(u, n) v_{p}(u, n)}{u^{2}}, \\
& v_{p}(u, n)=\sum_{d_{1} d_{2}=u} \cos \frac{2 \pi d_{1}}{p} \cdot d_{2}^{1-n}, v(u, n)=\sum_{d \mid u} d^{1-n}
\end{aligned}
$$

Proof. From Lemma 4, for $\chi(-1)=1$, we have

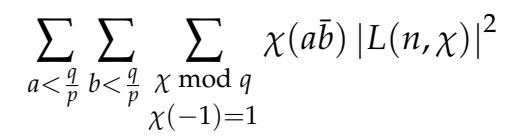

$$
\begin{aligned}
& =\sum_{\substack{\chi \bmod q \\
\chi(-1)=1}}|L(n, \chi)|^{2} \sum_{a<\frac{q}{p}} \chi(a) \sum_{b<\frac{q}{p}} \chi(\bar{b}) \\
& =\sum_{\substack{\chi \bmod q \\
\chi(-1)=1}}|L(n, \chi)|^{2} \frac{\tau(\chi)}{\pi i \phi(p)} \sum_{\substack{\xi \bmod p \\
\xi(-1)=-1}} \tau(\bar{\xi}) L(1, \xi \bar{\chi}) \frac{\tau(\bar{\chi})}{\pi i \phi(p)} \sum_{\substack{\lambda \bmod p \\
\lambda(-1)=-1}} \tau(\lambda) L(1, \bar{\lambda} \chi) \\
& =\frac{-q}{\pi^{2} \phi^{2}(p)} \sum_{\substack{\chi \bmod q \\
\chi(-1)=1}}|L(n, \chi)|^{2} \sum_{\substack{\xi \bmod p \\
\xi(-1)=-1}} \tau(\bar{\xi}) L(1, \xi \bar{\chi}) \sum_{\substack{\lambda \bmod p \\
\lambda(-1)=-1}} \tau(\lambda) L(1, \bar{\lambda} \chi) \\
& =\frac{-q}{\pi^{2} \phi^{2}(p)} \sum_{\substack{\chi \bmod q \\
\chi(-1)=1}} \sum_{\substack{\xi \bmod p \\
\xi(-1)=-1}} \tau(\bar{\xi}) \sum_{\substack{\lambda \bmod p \\
\lambda(-1)=-1}} \tau(\lambda) \\
& \times\left(\sum_{u=1}^{\infty} \frac{\bar{\chi}(u) \sum_{d_{1} d_{2}=u} \xi\left(d_{1}\right) d_{2}^{1-n}}{u}\right)\left(\sum_{v=1}^{\infty} \frac{\chi(v) \sum_{d_{1}^{\prime} d_{2}^{\prime}=v} \bar{\lambda}\left(d_{1}^{\prime}\right) d_{2}^{\prime 1-n}}{v}\right) \\
& :=\frac{-q}{\pi^{2} \phi^{2}(p)} M .
\end{aligned}
$$


For convenience, we put

$$
A(\bar{\chi}, \xi, y)=\sum_{N<u \leq y} \bar{\chi}(u) \sum_{d_{1} d_{2}=u} \xi\left(d_{1}\right) d_{2}^{1-n}
$$

where $N$ is a parameter with $q \leq N<q^{5}$. Then from Abel's identity we have

$$
\begin{aligned}
& M=\sum_{\substack{\chi \bmod q \\
\chi(-1)=1}} \sum_{\substack{\xi \bmod p \\
(-1)=-1}} \tau(\bar{\xi}) \sum_{\substack{\lambda \bmod p \\
\lambda(-1)=-1}} \tau(\lambda) \\
& \times\left(\sum_{u=1}^{\infty} \frac{\bar{\chi}(u) \sum_{d_{1} d_{2}=u} \xi\left(d_{1}\right) d_{2}^{1-n}}{u}\right)\left(\sum_{v=1}^{\infty} \frac{\chi(v) \sum_{d_{1}^{\prime} d_{2}^{\prime}=v} \bar{\lambda}\left(d_{1}^{\prime}\right) d_{2}^{\prime 1-n}}{v}\right) \\
& =\sum_{\substack{\chi \bmod q \\
\chi(-1)=1}} \sum_{\substack{\xi \bmod p \\
\xi(-1)=-1}} \tau(\bar{\xi}) \sum_{\substack{\lambda \bmod p \\
\lambda(-1)=-1}} \tau(\lambda) \\
& \times\left(\sum_{u \leq N} \frac{\bar{\chi}(u) \sum_{d_{1} d_{2}=u} \xi\left(d_{1}\right) d_{2}^{1-n}}{u}+\int_{N}^{\infty} \frac{A(\bar{\chi}, \xi, y)}{y^{2}} d y\right) \\
& \times\left(\sum_{v \leq N} \frac{\chi(v) \sum_{d_{1}^{\prime} d_{2}^{\prime}=v} \bar{\lambda}\left(d_{1}^{\prime}\right) d_{2}^{\prime 1-n}}{v}+\int_{N}^{\infty} \frac{A(\chi, \bar{\lambda}, y)}{y^{2}} d y\right) \\
& =\sum_{\substack{\chi \bmod q \\
\chi(-1)=1}} \sum_{\substack{\xi \bmod p \\
\xi(-1)=-1}} \tau(\bar{\xi}) \sum_{\substack{\lambda \bmod p \\
\lambda(-1)=-1}} \tau(\lambda) \\
& \times\left(\sum_{u \leq N} \frac{\bar{\chi}(u) \sum_{d_{1} d_{2}=u} \xi\left(d_{1}\right) d_{2}^{1-n}}{u}\right)\left(\sum_{v \leq N} \frac{\chi(v) \sum_{d_{1}^{\prime} d_{2}^{\prime}=v} \bar{\lambda}\left(d_{1}^{\prime}\right) d_{2}^{\prime 1-n}}{v}\right) \\
& +\sum_{\substack{\chi \bmod q \\
\chi(-1)=1}} \sum_{\substack{\xi \bmod p \\
\xi(-1)=-1}} \tau(\bar{\xi}) \sum_{\substack{\lambda \bmod p \\
\lambda(-1)=-1}} \tau(\lambda) \\
& \times\left(\sum_{u \leq N} \frac{\bar{\chi}(u) \sum_{d_{1} d_{2}=u} \xi\left(d_{1}\right) d_{2}^{1-n}}{u}\right)\left(\int_{N}^{\infty} \frac{A(\chi, \bar{\lambda}, y)}{y^{2}} d y\right) \\
& +\sum_{\substack{\chi \bmod q \\
\chi(-1)=1}} \sum_{\substack{\xi \bmod p \\
\xi(-1)=-1}} \tau(\bar{\xi}) \sum_{\substack{\lambda \bmod p \\
\lambda(-1)=-1}} \tau(\lambda) \\
& \times\left(\sum_{v \leq N} \frac{\chi(v) \sum_{d_{1}^{\prime} d_{2}^{\prime}=v} \bar{\lambda}\left(d_{1}^{\prime}\right) d_{2}^{\prime 1-n}}{v}\right)\left(\int_{N}^{\infty} \frac{A(\bar{\chi}, \xi, y)}{y^{2}} d y\right) \\
& +\sum_{\substack{\chi \bmod q \\
\chi(-1)=1 \xi(-1)=-1}} \sum_{\substack{\xi \bmod p \\
\lambda(-1)=-1}} \tau(\bar{\xi}) \sum_{\substack{\lambda \bmod p \\
\lambda(-1)}} \tau(\lambda)\left(\int_{N}^{\infty} \frac{A(\bar{\chi}, \xi, y)}{y^{2}} d y\right)\left(\int_{N}^{\infty} \frac{A(\chi, \bar{\lambda}, y)}{y^{2}} d y\right) \\
& :=M_{1}+M_{2}+M_{3}+M_{4} \text {, }
\end{aligned}
$$

we shall calculate each term in the above expression. 
(i) From Lemma 1 we have

$$
\begin{aligned}
\sum_{\substack{\chi \bmod q \\
\chi(-1)=1}} \chi(\bar{u} v) & =\frac{1}{2} \sum_{\chi \bmod q}(1+\chi(-1)) \chi(\bar{u} v) \\
& =\frac{1}{2} \sum_{\chi \bmod q} \chi(\bar{u} v)+\frac{1}{2} \sum_{\chi \bmod q} \chi(-\bar{u} v) \\
& =\frac{1}{2} \sum_{d \mid(q, \bar{u} v-1)} \mu\left(\frac{q}{d}\right) \phi(d)+\frac{1}{2} \sum_{d \mid(q, \bar{u} v+1)} \mu\left(\frac{q}{d}\right) \phi(d) .
\end{aligned}
$$

In addition, we have

$$
\begin{aligned}
\sum_{\substack{\xi \bmod p \\
\xi(-1)=-1}} \tau(\bar{\xi}) \xi\left(d_{1}\right) & =\sum_{\substack{\xi \bmod p \\
\xi(-1)=-1}} \sum_{a=1}^{p} \bar{\xi}(a) e^{2 \pi i \frac{a}{p}} \xi\left(d_{1}\right) \\
& =\sum_{\substack{\xi \bmod p \\
\xi(-1)=-1}} \sum_{a=1}^{p} \bar{\xi}(a) e^{2 \pi i \frac{a d_{1}}{p}} \\
& =\frac{\phi(p)}{2} e^{2 \pi i \frac{d_{1}}{p}}-\frac{\phi(p)}{2} e^{2 \pi i \frac{(p-1) d_{1}}{p}} \\
& =i \phi(p) \sin \frac{2 \pi d_{1}}{p} .
\end{aligned}
$$

Similarly, we can also get

$$
\sum_{\substack{\lambda \bmod p \\ \lambda(-1)=-1}} \tau(\lambda) \bar{\lambda}\left(d_{1}^{\prime}\right)=i \phi(p) \sin \frac{2 \pi d_{1}^{\prime}}{p} .
$$

So, we have

$$
\begin{aligned}
& \sum_{\substack{\xi \bmod p \\
\xi(-1)=-1}} \tau(\bar{\xi}) \sum_{\substack{\lambda \bmod p \\
\lambda(-1)=-1}} \tau(\lambda) \sum_{d_{1} d_{2}=u} \xi\left(d_{1}\right) d_{2}^{1-n} \sum_{d_{1}^{\prime} d_{2}^{\prime}=v} \bar{\lambda}\left(d_{1}^{\prime}\right) d_{2}^{\prime 1-n} \\
= & -\phi^{2}(p) \sum_{d_{1} d_{2}=u} \sin \frac{2 \pi d_{1}}{p} \cdot d_{2}^{1-n} \sum_{d_{1}^{\prime} d_{2}^{\prime}=v} \sin \frac{2 \pi d_{1}^{\prime}}{p} \cdot d_{2}^{\prime 1-n} \\
= & -\phi^{2}(p) \gamma_{p}(u, n) \gamma_{p}(v, n) .
\end{aligned}
$$

Hence, we can write

$$
\begin{aligned}
M_{1}= & \sum_{\substack{\chi \bmod q \\
\chi(-1)=1 \xi(-1)=-1}} \sum_{\substack{\xi \bmod p \\
\lambda(-1)=-1}} \tau(\bar{\xi}) \sum_{\substack{\bmod p \\
\xi(\lambda)}} \tau(\lambda) \\
& \times\left(\sum_{u \leq N} \frac{\bar{\chi}(u) \sum_{d_{1} d_{2}=u} \xi\left(d_{1}\right) d_{2}^{1-n}}{u}\right)\left(\sum_{v \leq N} \frac{\left.\chi(v) \sum_{d_{1}^{\prime} d_{2}^{\prime}=v} \bar{\lambda}\left(d_{1}^{\prime}\right) d_{2}^{\prime 1-n}\right)}{v}\right) \\
= & \sum_{\substack{\xi \bmod p \\
\xi(-1)=-1}} \tau(\bar{\xi}) \sum_{\substack{\lambda \bmod p \\
\lambda(-1)=-1}} \tau(\lambda)
\end{aligned}
$$




$$
\begin{aligned}
& \times \sum_{1 \leq u \leq N}^{\prime} \sum_{1 \leq v \leq N}^{\prime} \frac{\sum_{d_{1} d_{2}=u} \xi\left(d_{1}\right) d_{2}^{1-n} \sum_{d_{1}^{\prime} d_{2}^{\prime}=v} \bar{\lambda}\left(d_{1}^{\prime}\right) d_{2}^{\prime 1-n}}{u v} \sum_{\substack{\chi \bmod q \\
\chi(-1)=1}} \chi(\bar{u} v) \\
& =-\frac{1}{2} \sum_{1 \leq u \leq N}^{\prime} \sum_{1 \leq v \leq N}^{\prime} \frac{\phi^{2}(p) \gamma_{p}(u, n) \gamma_{p}(v, n)}{u v} \sum_{d \mid(q, \bar{u} v-1)} \mu\left(\frac{p}{d}\right) \phi(d) \\
& -\frac{1}{2} \sum_{1 \leq u \leq N}^{\prime} \sum_{1 \leq v \leq N}^{\prime} \frac{\phi^{2}(p) \gamma_{p}(u, n) \gamma_{p}(v, n)}{u v} \sum_{d \mid(q, \bar{u} v+1)} \mu\left(\frac{q}{d}\right) \phi(d) \\
& =-\frac{1}{2} \sum_{d \mid q} \mu\left(\frac{q}{d}\right) \phi(d) \sum_{\substack{1 \leq u \leq N \\
u \equiv v(\bmod d)}}^{\prime} \sum_{\substack{1 \leq v \leq N \\
\prime}} \frac{\phi^{2}(p) \gamma_{p}(u, n) \gamma_{p}(v, n)}{u v} \\
& -\frac{1}{2} \sum_{d \mid q} \mu\left(\frac{q}{d}\right) \phi(d) \sum_{\substack{1 \leq u \leq N \\
u \equiv-v(\bmod d)}}^{\prime} \sum_{\substack{1 \leq v \leq N \\
u}}^{\prime} \frac{\phi^{2}(p) \gamma_{p}(u, n) \gamma_{p}(v, n)}{u v}
\end{aligned}
$$

where $\sum_{1 \leq n \leq N}^{\prime}$ denotes the summation over $n$ from 1 to $N$ such that $(n, q)=1$.

For calculation convenience, we divide the sum over $u$ or $v$ into four cases: (i) $d \leq u, v \leq N$; (ii) $d \leq u \leq N$ and $1 \leq v \leq d-1$; (iii) $1 \leq u \leq d-1$ and $d \leq v \leq N$; iv) $1 \leq u, v \leq d-1$. So we have

$$
\begin{aligned}
& \sum_{d \mid q} \mu\left(\frac{q}{d}\right) \phi(d) \sum_{\substack{d \leq u \leq N \\
u \equiv v(\bmod d)}}^{\prime} \sum_{\substack{d \leq v \leq N \\
\text { mod }}} \frac{\phi^{2}(p) \gamma_{p}(u, n) \gamma_{p}(v, n)}{u v} \\
& \ll \sum_{d \mid q} \phi(d) \sum_{1 \leq r_{1} \leq \frac{N}{d}} \sum_{1 \leq r_{2} \leq \frac{N}{d}} \sum_{\substack{l_{1}=1 \\
l_{1} \equiv l_{2}(\bmod d)}}^{d-1} \sum_{l_{1}=1}^{d-1} \frac{\phi^{2}(p) \gamma_{p}\left(r_{1} d+l_{1}, n\right) \gamma_{p}\left(r_{2} d+l_{2}, n\right)}{\left(r_{1} d+l_{1}\right)\left(r_{2} d+l_{2}\right)} \\
& \ll \phi^{2}(p) \sum_{d \mid q} \phi(d) \sum_{1 \leq r_{1} \leq \frac{N}{d}} \sum_{1 \leq r_{2} \leq \frac{N}{d}} \sum_{l_{1}=1}^{d-1} \frac{\sum_{d_{2} \mid\left(r_{1} d+l_{1}\right)} d_{2}^{1-n} \sum_{d_{2}^{\prime} \mid\left(r_{2} d+l_{1}\right)} d_{2}^{\prime 1-n}}{\left(r_{1} d+l_{1}\right)\left(r_{2} d+l_{1}\right)} \\
& \ll \phi^{2}(p) \sum_{d \mid q} \phi(d) \sum_{1 \leq r_{1} \leq \frac{N}{d}} \sum_{1 \leq r_{2} \leq \frac{N}{d}} \sum_{l_{1}=1}^{d-1} \frac{\tau_{2}\left(r_{1} d+l_{1}\right) \tau_{2}\left(r_{2} d+l_{1}\right)}{\left(r_{1} d+l_{1}\right)\left(r_{2} d+l_{1}\right)} \\
& \ll \phi^{2}(p) \sum_{d \mid q} \frac{\phi(d)}{d} \sum_{1 \leq r_{1} \leq \frac{N}{d}} \sum_{1 \leq r_{2} \leq \frac{N}{d}} \frac{\left[\left(r_{1} d+1\right)\left(r_{2} d+1\right)\right]^{\epsilon}}{r_{1} r_{2}} \\
& \ll \phi^{2}(p) q^{\epsilon} .
\end{aligned}
$$

$$
\begin{aligned}
& \sum_{d \mid q} \mu\left(\frac{q}{d}\right) \phi(d) \sum_{\substack{d \leq u \leq N \\
u \equiv v(\bmod d)}}^{\prime} \sum_{\substack{1 \leq v \leq d-1 \\
u}}^{\prime} \frac{\phi^{2}(p) \gamma_{p}(u, n) \gamma_{p}(v, n)}{u v} \\
& \ll \sum_{d \mid q} \phi(d) \sum_{1 \leq r_{1} \leq \frac{N}{d}} \sum_{\substack{1 \leq v \leq d-1 \\
v \equiv l_{1}(\bmod d)}}^{\sum_{l_{1}=1}^{d-1}} \frac{\phi^{2}(p) \gamma_{p}\left(r_{1} d+l_{1}, n\right) \gamma_{p}(v, n)}{\left(r_{1} d+l_{1}\right) v} \\
& \ll \quad \phi^{2}(p) \sum_{d \mid q} \phi(d) \sum_{\substack{1 \leq r_{1} \leq \frac{N}{d} \\
1 \leq v \leq d-1}} \sum_{\substack{\sum_{1 \leq r_{1} \leq \frac{N}{d}} \\
\sum^{2}(p) \sum_{d \mid q} \phi(d \leq d-1}}\left(r_{1} v d\right)^{\epsilon-1}
\end{aligned}
$$




$$
\ll \phi^{2}(p) q^{\epsilon} .
$$

and

$$
\begin{aligned}
& \sum_{d \mid q} \mu\left(\frac{q}{d}\right) \phi(d) \sum_{\substack{1 \leq u \leq d-1 \\
u \equiv v(\bmod d)}}^{\prime} \sum_{\substack{d \leq v \leq N \\
\text { (n) }}} \frac{\phi^{2}(p) \gamma_{p}(u, n) \gamma_{p}(v, n)}{u v} \\
\ll & \phi^{2}(p) \sum_{d \mid q} \phi(d) \sum_{\substack{1 \leq u \leq d-1 \\
1 \leq r_{2} \leq \frac{N}{d}}}\left(u r_{2} d\right)^{\epsilon-1} \\
\ll & \phi^{2}(p) q^{\epsilon},
\end{aligned}
$$

where we have used the estimate $\tau_{2}(n) \ll n^{\epsilon}$.

For the case $1 \leq u, v \leq d-1$, the solution of the congruence $u \equiv v(\bmod d)$ is $u=v$. Hence,

$$
\begin{aligned}
& \sum_{d \mid q} \mu\left(\frac{q}{d}\right) \phi(d) \sum_{\substack{1 \leq u \leq d-1 \\
u \equiv v(\bmod d)}}^{\prime} \sum_{1 \leq v \leq d-1}^{\prime} \frac{\phi^{2}(p) \gamma_{p}(u, n) \gamma_{p}(v, n)}{u v} \\
= & \phi^{2}(p) \sum_{d \mid q} \mu\left(\frac{q}{d}\right) \phi(d) \sum_{\substack{1 \leq u \leq d-1 \\
\prime}} \frac{\gamma_{p}^{2}(u, n)}{u^{2}} \\
= & \phi^{2}(p) \sum_{d \mid q} \mu\left(\frac{q}{d}\right) \phi(d) \sum_{\substack{u=1 \\
(u, q)=1}}^{\infty} \frac{\gamma_{p}^{2}(u, n)}{u^{2}}+O\left(\phi^{2}(p) q^{\epsilon}\right) \\
= & (q-2) \phi^{2}(p) \sum_{\substack{u=1 \\
(u, q)=1}}^{\infty} \frac{\gamma_{p}^{2}(u, n)}{u^{2}}+O\left(\phi^{2}(p) q^{\epsilon}\right) \\
= & (q-2) \phi^{2}(p)\left(\sum_{u=1}^{\infty} \frac{\gamma_{p}^{2}(u, n)}{u^{2}}-\sum_{u=1}^{\infty} \frac{\gamma_{p}^{2}(u, n)}{u^{2}}\right)+O\left(\phi^{2}(p) q^{\epsilon}\right) \\
= & (q-2) \phi^{2}(p) \sum_{u=1}^{\infty} \frac{\gamma_{p}^{2}(u, n)}{u^{2}}+O\left(\phi^{2}(p) q^{\epsilon}\right) .
\end{aligned}
$$

Then from (3)-(6), we have

$$
\begin{aligned}
& -\frac{1}{2} \sum_{d \mid q} \mu\left(\frac{q}{d}\right) \phi(d) \sum_{\substack{1 \leq u \leq N \\
u \equiv v(\bmod d)}}^{\prime} \sum_{\substack{1 \leq v \leq N \\
\prime}}^{\prime} \frac{\phi^{2}(p) \gamma_{p}(u, n) \gamma_{p}(v, n)}{u v} \\
= & -\frac{\phi^{2}(p)(q-2)}{2} \sum_{u=1}^{\infty} \frac{\gamma_{p}^{2}(u, n)}{u^{2}}+O\left(\phi^{2}(p) q^{\epsilon}\right) \\
= & -\frac{\phi^{2}(p)(q-2)}{2} C_{p, n}+O\left(\phi^{2}(p) q^{\epsilon}\right) .
\end{aligned}
$$

Similarly, we can also get the estimate

$$
\begin{gathered}
-\frac{1}{2} \sum_{d \mid q} \mu\left(\frac{q}{d}\right) \phi(d) \sum_{\substack{1 \leq u \leq N \\
u \equiv-v(\bmod d)}}^{\prime} \sum_{\substack{1 \leq v \leq N \\
\text { mod }}}^{\prime} \frac{\phi^{2}(p) \gamma_{p}(u, n) \gamma_{p}(v, n)}{u v} \\
=-\frac{1}{2} \sum_{d \mid q} \mu\left(\frac{q}{d}\right) \phi(d) \sum_{\substack{1 \leq u \leq N \\
1 \leq v \leq N \\
u+v=d}}^{\prime} \sum_{\substack{1 \leq v \leq 1 \\
\text { d }}}^{\prime} \frac{\phi^{2}(p) \gamma_{p}(u, n) \gamma_{p}(v, n)}{u v}
\end{gathered}
$$




$$
\begin{aligned}
& \quad-\frac{1}{2} \sum_{d \mid q} \mu\left(\frac{q}{d}\right) \phi(d) \sum_{\substack{1 \leq u \leq N \\
u+v=l d, l \geq 2}}^{\prime} \sum_{1 \leq v \leq N}^{\prime} \frac{\phi^{2}(p) \gamma_{p}(u, n) \gamma_{p}(v, n)}{u v} \\
& \ll \quad \phi^{2}(p) \sum_{d \mid q} \phi(d) \sum_{1 \leq u \leq d-1} \frac{\gamma_{p}(u, n) \gamma_{p}(d-u, n)}{u(d-u)} \\
& +\phi^{2}(p) \sum_{d \mid q} \phi(d) \sum_{1 \leq u \leq N}^{\prime} \sum_{l=\left[\frac{u}{d}\right]+2}^{\left[\frac{N+u}{d}\right]} \frac{\gamma_{p}(u, n) \gamma_{p}(l d-u, n)}{l d u-u^{2}} \\
& \ll \quad \phi^{2}(p) \sum_{d \mid q} \frac{\phi(d)}{d} \sum_{1 \leq u \leq d-1} \frac{u^{\epsilon}(d-u)^{\epsilon}}{u} \\
& \quad+\phi^{2}(p) \sum_{d \mid q} \frac{\phi(d)}{d} \sum_{1 \leq u \leq N} \sum_{l=\left[\frac{u}{d}\right]+2}^{\left[\frac{N+u}{d}\right]} \frac{u^{\epsilon}(l d-u)^{\epsilon}}{l u-\frac{u^{2}}{d}} \\
& \ll \quad \phi^{2}(p)\left(q^{\epsilon}+\sum_{d \mid q} \frac{\phi(d)}{d} \sum_{u=1}^{N} \sum_{l=1}^{N} \frac{u^{\epsilon} l^{\epsilon}}{u l}\right) \\
& \ll \quad \phi^{2}(p) q^{\epsilon} .
\end{aligned}
$$

Then combining (2), (7) and (8), we have

$$
M_{1}=-\frac{\phi^{2}(p)(q-2)}{2} C_{p, n}+O\left(\phi^{2}(p) q^{\epsilon}\right)
$$

(ii) From Lemma 4 of [15], we have the estimate

$$
\sum_{\chi \neq \chi_{0}}|A(y, \chi)|^{2} \ll y^{1+\epsilon} q^{2}
$$

where $\chi_{0}$ denotes the principal character modulo $q, A(y, \chi)=\sum_{N<n \leq y} \chi(n) \tau_{2}(n)$. Then from the Cauchy inequality we can easily get

$$
\sum_{\chi(-1)=1}|A(y, \chi)| \ll \sum_{\chi \neq \chi_{0}}|A(y, \chi)| \ll y^{\frac{1}{2}+\epsilon} q^{\frac{3}{2}} .
$$

Using this estimate we have

$$
\begin{aligned}
M_{2}= & \sum_{\substack{\chi \bmod q \\
\chi(-1)=1}} \sum_{\substack{\xi \bmod p \\
\xi(-1)=-1}} \tau(\bar{\xi}) \sum_{\substack{\lambda \bmod p \\
\lambda(-1)=-1}} \tau(\lambda) \\
& \times\left(\sum_{u \leq N} \frac{\bar{\chi}(u) \sum_{d_{1} d_{2}=u} \bar{\xi}\left(d_{1}\right) d_{2}^{1-n}}{u}\right)\left(\int_{N}^{\infty} \frac{A(\chi, \bar{\lambda}, y)}{y^{2}} d y\right) \\
= & -\phi^{2}(p) \sum_{1 \leq u \leq N} \frac{\bar{\chi}(u) \gamma_{p}(u, n)}{u} \int_{N}^{\infty} \frac{1}{y^{2}}\left(\sum_{\chi \bmod q} \sum_{\chi<v \leq y} \chi(v) \gamma_{k}(v, n)\right) d y \\
\ll & \phi^{2}(p) \sum_{1 \leq u \leq N} \frac{\bar{\chi}(u) d(u)}{u} \int_{N}^{\infty} \frac{1}{y^{2}}\left(\sum_{\substack{\bmod q \\
\chi(-1)=1}} A(y, \chi)\right) d y
\end{aligned}
$$




$$
\begin{aligned}
& \ll \phi^{2}(p) \sum_{1 \leq u \leq N} u^{\epsilon-1} \int_{N}^{\infty} \frac{1}{y^{2}}\left(\sum_{\substack{\chi \bmod q \\
\chi(-1)=1}}|A(y, \chi)|\right) d y \\
& \ll \phi^{2}(p) N^{\epsilon} \int_{N}^{\infty} \frac{q^{\frac{3}{2}} y^{\frac{1}{2}+\epsilon_{1}}}{y^{2}} d y \ll \phi^{2}(p) \frac{q^{\frac{3}{2}}}{N^{\frac{1}{2}-\epsilon}} .
\end{aligned}
$$

(iii) Similar to (ii), we can also get

$$
M_{3} \ll \phi^{2}(p) \frac{q^{\frac{3}{2}}}{N^{\frac{1}{2}-\epsilon}} .
$$

(iv) Using the same discussion in (ii), and making use of the absolute convergent properties of the integral, we can calculate

$$
\begin{aligned}
M_{4} & =\sum_{\substack{\chi \bmod q \\
\chi(-1)=1}} \sum_{\substack{\xi(-1)=-1 \\
\text { mod } p}} \tau(\bar{\xi}) \sum_{\substack{\lambda \bmod p \\
\lambda(-1)=-1}} \tau(\lambda)\left(\int_{N}^{\infty} \frac{A(\bar{\chi}, \xi, y)}{y^{2}} d y\right)\left(\int_{N}^{\infty} \frac{A(\chi, \bar{\lambda}, y)}{y^{2}} d y\right) \\
& =\phi^{2}(p) \sum_{\substack{\chi \bmod q \\
\chi(-1)=1}}\left(\int_{N}^{\infty} \frac{1}{y^{2}} \sum_{N<u \leq y} \bar{\chi}(u) \gamma_{p}(u, n) d y\right)\left(\int_{N}^{\infty} \frac{1}{y^{2}} \sum_{N<v \leq y} \chi(v) \gamma_{p}(v, n) d y\right) \\
& \ll \phi^{2}(p) \sum_{\chi \bmod p}\left(\int_{N}^{\infty} \frac{A(y, \bar{\chi})}{y^{2}} d y\right)\left(\int_{N}^{\infty} \frac{A(y, \chi)}{y^{2}} d y\right) \\
& \leq \phi^{2}(p) \int_{N}^{\infty} \int_{N}^{\infty} \frac{1}{y^{2} z^{2}} \sum_{\chi \bmod q}|A(y, \bar{\chi})||A(y, \chi)| d y d z \\
& \ll \phi^{2}(p) \int_{N}^{\infty} \frac{1}{y^{2}} \int_{N}^{\infty} \frac{1}{z^{2}}\left(\sum_{\chi \neq \chi_{0}}|A(y, \bar{\chi})|^{2}\right)^{\frac{1}{2}}\left(\sum_{\chi \neq \chi_{0}}|A(y, \chi)|^{2}\right)^{\frac{1}{2}} d y d z \\
& \ll \phi^{2}(p)\left(\int_{N}^{\infty} \frac{1}{y^{2}}\left(\sum_{\chi \neq \chi_{0}}|A(y, \chi)|^{2}\right)^{\frac{1}{2}} d y\right)^{2} \\
& \ll \phi^{2}(p)\left(\int_{N}^{\infty} \frac{q}{y^{\frac{3}{2}-\epsilon}} d y\right)^{2} \ll \phi^{2}(p) \frac{q^{2}}{N^{1-\epsilon}} .
\end{aligned}
$$

Now taking $N=q^{4}$ and $\epsilon<\frac{1}{2}$, combining (1) and (9)-(12), we have

$$
M=-\frac{\phi^{2}(p)(q-2)}{2} C_{p, n}+O\left(\phi^{2}(p) q^{\epsilon}\right)
$$

Thus we obtain the asymptotic formula for $\chi(-1)=1$,

$$
\sum_{a<\frac{q}{p}} \sum_{b<\frac{q}{p}} \sum_{\substack{\chi \bmod q \\ \chi(-1)=1}} \chi(a \bar{b})|L(n, \chi)|^{2}=\frac{-q}{\pi^{2} \phi^{2}(p)} M=\frac{q^{2}}{2 \pi^{2}} C_{p, n}+O\left(q^{1+\epsilon}\right) .
$$


For $\chi(-1)=-1$, from Lemma 4 , we have

$$
\begin{aligned}
& \sum_{a<\frac{q}{p}} \sum_{b<\frac{q}{p}} \sum_{\chi \bmod q} \chi(a \bar{b})|L(n, \chi)|^{2} \\
& =\sum_{\substack{\chi \bmod q \\
\chi(-1)=-1}}|L(n, \chi)|^{2}\left[\frac{\tau(\chi)}{\pi i}\left(1-\frac{\bar{\chi}(p)}{p}\right) L(1, \bar{\chi})+\frac{i \tau(\chi)}{\pi \phi(p)} \sum_{\substack{\xi \bmod p \\
\xi(-1)=1}} \tau(\bar{\xi}) L(1, \bar{\xi} \bar{\chi})\right] \\
& \times\left[\frac{\tau(\bar{\chi})}{\pi i}\left(1-\frac{\chi(p)}{p}\right) L(1, \chi)+\frac{i \tau(\bar{\chi})}{\pi \phi(p)} \sum_{\substack{\lambda \bmod p \\
\lambda(-1)=1}} \tau(\lambda) L(1, \bar{\lambda} \chi)\right] \\
& =\frac{q}{\pi^{2}} \sum_{\substack{\chi \bmod q \\
\chi(-1)=-1}}\left(1-\frac{\bar{\chi}(p)}{p}\right)\left(1-\frac{\chi(p)}{p}\right)|L(n, \chi)|^{2}|L(1, \chi)|^{2} \\
& +\frac{-q}{\pi^{2} \phi(p)} \sum_{\substack{\chi \bmod q \\
\chi(-1)=-1}}\left(1-\frac{\bar{\chi}(p)}{p}\right)|L(n, \chi)|^{2} L(1, \bar{\chi}) \sum_{\substack{\lambda \bmod p \\
\lambda(-1)=1}} \tau(\lambda) L(1, \bar{\lambda} \chi) \\
& +\frac{-q}{\pi^{2} \phi(p)} \sum_{\substack{\chi \bmod q \\
\chi(-1)=-1}}\left(1-\frac{\chi(p)}{p}\right)|L(n, \chi)|^{2} L(1, \chi) \sum_{\substack{\xi \bmod p \\
\xi(-1)=1}} \tau(\bar{\xi}) L(1, \xi \bar{\chi}) \\
& +\frac{q}{\pi^{2} \phi^{2}(p)} \sum_{\substack{\chi \bmod q \\
\chi(-1)=-1}}|L(n, \chi)|^{2} \sum_{\substack{\xi \bmod p \\
\xi(-1)=1}} \tau(\bar{\xi}) L(1, \xi \bar{\chi}) \sum_{\substack{\lambda \bmod p \\
\lambda(-1)=1}} \tau(\lambda) L(1, \bar{\lambda} \chi) \\
& :=A+B+C+D \text {. }
\end{aligned}
$$

Using the same method as proving $\chi(-1)=1$, we can easily get

$$
\begin{gathered}
A=\frac{q^{2}}{2 \pi^{2}}\left(1+\frac{1}{p^{2}}\right) \sum_{u=1}^{\infty} \frac{v^{2}(u, n)}{u^{2}}-\frac{q^{2}}{\pi^{2} p^{2}} \sum_{u=1}^{\infty} \frac{v(u, n) v(p u, n)}{u^{2}}+O\left(q^{1+\epsilon}\right), \\
B=C=\frac{q^{2}}{2 \pi^{2} p^{2}} \sum_{u=1}^{\infty} \frac{v(u, n) v_{p}(p u, n)}{u^{2}}-\frac{p^{2}}{2 \pi^{2}} \sum_{u=1}^{\infty} \frac{v(u, n) v_{p}(u, n)}{u^{2}}+O\left(q^{1+\epsilon}\right), \\
D=\frac{q^{2}}{2 \pi^{2}} \sum_{u=1}^{\infty} \frac{v_{p}^{2}(u, n)}{u^{2}}+O\left(q^{1+\epsilon}\right) .
\end{gathered}
$$

Thus we obtain the asymptotic formula for $\chi(-1)=-1$,

$$
\sum_{a<\frac{q}{p}} \sum_{b<\frac{q}{p}} \sum_{\substack{\chi \bmod q \\ \chi(-1)=-1}} \chi(a \bar{b})|L(n, \chi)|^{2}=\frac{q^{2}}{\pi^{2}} T_{p, n}+O\left(q^{1+\epsilon}\right) .
$$

This proves Lemma 5.

\section{Proof of Theorem and Corollaries}

In this section we will accomplish the proof of the theorem and corollaries. From Lemmas 2 and 5 , we have: 
(i) when $n$ be an even number,

$$
\begin{aligned}
& \sum_{a<\frac{q}{p}} \sum_{b<\frac{q}{p}} S(a \bar{b}, n, q) \\
= & \frac{(n !)^{2} q}{4^{n-1} \pi^{2 n} \phi(q)} \sum_{a<\frac{q}{p}} \sum_{b<<\frac{q}{p}} \sum_{\chi \bmod q} \chi(a \bar{b})|L(n, \chi)|^{2}+\frac{(n !)^{2} \zeta^{2}(n)}{4^{n-1} \pi^{2 n}}\left(\frac{1}{q^{2 n-1}}-1\right) \sum_{a<\frac{q}{p}} \sum_{b<\frac{q}{p}} 1 \\
= & \frac{(n !)^{2} q^{2}}{2^{2 n-2} \pi^{2 n}}\left(\frac{1}{2 \pi^{2}} C_{p, n}-\frac{\zeta^{2}(n)}{p^{2}}\right)+O\left(q^{1+\epsilon}\right) .
\end{aligned}
$$

(ii) when $n$ be an odd number,

$$
\begin{aligned}
\sum_{a<\frac{q}{p}} \sum_{b<\frac{q}{p}} S(a \bar{b}, n, q) & =\frac{(n !)^{2} q}{4^{n-1} \pi^{2 n} \phi(q)} \sum_{a<\frac{q}{p}} \sum_{b<\frac{q}{p}} \sum_{\substack{\chi \bmod q \\
\chi(-1)=-1}} \chi(a \bar{b})|L(n, \chi)|^{2} \\
& =\frac{(n !)^{2} q}{4^{n-1} \pi^{2 n} \phi(q)}\left(\frac{q^{2}}{\pi^{2}} T_{p, n}+O\left(q^{1+\epsilon}\right)\right) \\
& =\frac{(n !)^{2} q^{2}}{2^{2 n-2} \pi^{2 n+2}} T_{p, n}+O\left(q^{1+\epsilon}\right) .
\end{aligned}
$$

This completes the proof of the Theorem.

Taking $p=2$ and $n=2$ or 4 in the Theorem, we get $C_{2,2}=C_{2,4}=0$. When $n$ be an even number, from reference [7], we can easily calculate

$$
S_{n}^{(1)}(h, k)=\frac{(2 \pi)^{n-1}}{i^{n+1} \cdot n !} S(h, n, k) .
$$

Noting that $\zeta(2)=\pi^{2} / 6$ and $\zeta(4)=\pi^{4} / 90$, we easily get Corollarys 1 and 2 .

Author Contributions: Conceptualization, L.L. and Z.X.; methodology, L.L. and Z.X.; software, L.L. and Z.X.; validation, L.L. and Z.X.; formal analysis, L.L. and Z.X.; investigation, L.L. and Z.X.; resources, L.L. and Z.X.; data curation, L.L. and Z.X.; writing-original draft preparation, L.L. and Z.X.; writing-review and editing, L.L. and Z.X.; visualization, L.L. and Z.X.; supervision, L.L. and Z.X.; project administration, L.L. and Z.X.; funding acquisition, L.L. and Z.X. All authors have read and agreed to the published version of the manuscript.

Funding: This work is supported by the Basic Research Program for Nature Science of the Shaanxi Province (2014JM1001, 2015KJXX-27) and N.S.F. (11971381, 11471258, 11701447) of China.

Conflicts of Interest: The authors declare no conflict of interest.

\section{References}

1. Carlitz, L. The reciprocity theorem of Dedekind sums. Pac. J. Math. 1953, 3, 513-522. [CrossRef]

2. Conrey, J.B.; Fransen, E.; Klein, R.; Scott, C. Mean values of Dedekind sums. J. Number Theory 1996, 41, 214-226. [CrossRef]

3. Jia, C. On the mean values of Dedekind sums. J. Number Theory 2001, 87, 173-188. [CrossRef]

4. Zhang, W. On the mean values of Dedekind sums. J. Theor. Nombres Bordx. 1996, 8, 429-442. [CrossRef]

5. Zhang, W. A note on the mean square value of the Dedekind sums. Acta Math. Hung. 2000, 86, $275-289$. [CrossRef]

6. Zhang, W.; Yi, Y. Partial sums of Dedekind sums. Prog. Nat. Sci. 2000, 4, 314-319.

7. Zhang, W. On the general Dedekind sums and one kind identities of Dirichlet L-functions. Acta Math. Sin. 2001, 44, 269-272.

8. Kim, T.; San Kim, D.; Lee, H.; Jang, L.C. Identities on poly-dedekind sums. Adv. Differ. Equations 2020, 1, $1-13$.

9. Ma, Y.; San Kim, D.; Lee, H.; Kim, T. Poly-Dedekind sums associated with poly-Bernoulli functions. J. Inequalities Appl. 2020, 248, 1-10. 
10. Kim, T. Note on q-dedekind type sums related to q-euler polynomials. Glasg. Math. J. 2012, 54, 121-125. [CrossRef]

11. Shparlinski, I.E. On some weighted average value of L-functions. Bull. Aust. Math. Soc 2009, 79, $183-186$. [CrossRef]

12. Liu, L.; Xu, Z.; Wang, N. Mean values of generalized Dedekind sums over short intervals. Acta Arith. 2020, 193, 95-108. [CrossRef]

13. Zhang, W. On a Cochrane sum and its hybrid mean value formula(II). J. Math. Appl. 2002, 276, $446-457$. [CrossRef]

14. Peral, C.J. Character sums and explicit estimates for L-functions. Contemp. Math. 1995, 189, 449-459.

15. Zhang, W.; Yi, Y.; He, X. On the $2 k$-th power mean of Dirichlet L-functions with the weight of general Kloosterman sums. J. Number Theory 2000, 84, 199-213.

Publisher's Note: MDPI stays neutral with regard to jurisdictional claims in published maps and institutional affiliations.

(C) 2020 by the authors. Licensee MDPI, Basel, Switzerland. This article is an open access article distributed under the terms and conditions of the Creative Commons Attribution (CC BY) license (http://creativecommons.org/licenses/by/4.0/). 